\title{
Transient and pretransient stages in a field induced phase transition of the vacuum state
}

\author{
Chitradip Banerjee ${ }^{1,2, *}$ and Manoranjan P. Singh ${ }^{1,2}$ \\ ${ }^{1}$ Theory and Simulations Lab, HRDS, Raja Ramanna Centre for Advanced Technology, \\ Indore 452013, India \\ ${ }^{2}$ Homi Bhabha National Institute, Training School Complex, Anushakti Nagar, Mumbai 400094, India
}

(Received 19 September 2018; revised manuscript received 14 July 2019; published 23 September 2019)

\begin{abstract}
The evolution of the modulus and phase of the complex order parameter associated with the fieldinduced phase transition (FIPT) of the vacuum state interacting with a time-dependent Sauter pulse is studied to analyze different evolution stages of the order parameter: e.g., the quasielectron-positron plasma (QEPP), transient, and residual electron-positron plasma (REPP) stages. By revisiting FIPT in the presence of single-sheeted and multisheeted pulses, we attribute the transient stage to the nonlinear coupling in the differential equations governing the dynamics of the phase and the modulus of the order parameter. The appearance of rapid oscillations in the modulus is shown to be associated with the abrupt change in the phase of the order parameter in the transient stage. FIPT is also studied for a multisheeted Sauter pulse with linear and quadratic frequency chirp. The QEPP stage is found to show complex dynamical behavior with fast and irregular oscillations due to the frequency chirp. The formation of the pretransient region due to the quadratic frequency chirping is observed in the accelerating part of the QEPP stage before the electric field attains the maximum value. As the quadratic chirp is increased, the pretransient and transient stages move closer to the electric field maximum, which leads to a decrease in temporal separation between the two stages. The early appearance of the transient stage, and hence of the following REPP stage, results in the enhancement of the pair production rate.
\end{abstract}

DOI: $10.1103 /$ PhysRevD.100.056016

\section{INTRODUCTION}

Electron-positron pair production from an unstable vacuum in the presence of a uniform electric field, widely known as the Schwinger mechanism [1-5], is one of the intriguing phenomena in the nonperturbative regime of quantum electrodynamics (QED). This process is suppressed exponentially for the electric field strength $E \ll E_{S}$, where the critical electric field of QED $E_{S}=$ $m^{2} c^{3} / e \hbar=1.38 \times 10^{18} \mathrm{~V} / \mathrm{m}$ [2], and the probability of pair production in the presence of a constant electric field $E$ is given by $P_{e^{+} e^{-}} \approx \exp \left(-\pi E_{S} / E\right)$. As $E \ll E_{S}$ for state-ofthe-art present-day ultraintense lasers and other light sources, this fundamental prediction of QED could not be tested experimentally. However, recent advances in laser technology, especially the use of the chirp pulse amplification method, promise to fast narrow the gap [6].

\footnotetext{
*Corresponding author. banerjee.chitradip@gmail.com

Published by the American Physical Society under the terms of the Creative Commons Attribution 4.0 International license. Further distribution of this work must maintain attribution to the author(s) and the published article's title, journal citation, and DOI. Funded by SCOAP.
}

The European Extreme Light Infrastructure for Nuclear Physics (ELI-NP) is planning to build a $10 \mathrm{PW}$ pulsed laser to achieve intensities $I \sim 10^{23} \mathrm{~W} / \mathrm{cm}^{2}$ for the first time for investigating new physical phenomena at the interfaces of plasma, nuclear, and particle physics [7-10]. The electric field at the laser focus will have a maximum value of $10^{15} \mathrm{~V} / \mathrm{m}$ at such intensities. In the ELI-NP experimental area E6, there is a proposal to study radiation reactions, strong-field QED effects, and the resulting production of ultrabright gamma rays which could be used for nuclear activation.

The construction of an x-ray free electron laser (XFEL) based on the principle of self-amplified spontaneous emission (SASE) is underway at DESY, Hamburg [11], which may produce a subcritical electric field. This facility may have the potential to generate a considerable number of electron-positron pairs.

In a landmark experiment E144 at the Stanford Linac Acceleration Center (SLAC) in 1997, it was possible to observe nonlinear QED processes like nonlinear Compton scattering and stimulated pair production in the collision of a $46.6 \mathrm{GeV}$ electron beam with a laser beam of intensity $I=1.3 \times 10^{18} \mathrm{~W} / \mathrm{cm}^{2}[12,13]$. Although these processes pertain to the perturbative regime of QED, the successful experimental realizations thereof raise hope for the 
experimental verification of the Schwinger mechanism in coming decades.

The original formalism of the Schwinger mechanism was developed for an electric field constant in space and time. It was generalized subsequently for a time-varying field [14] and is applied to study pair production by ultraintense and ultrashort laser pulses with the consideration that the length and timescales of the variation in the field are much less than the Compton length and time. As the pulse duration of some of the proposed light sources (e.g., lasers) based on higher-order harmonics is expected to be of the order of attoseconds, one needs to look beyond the Schwinger formalism. Furthermore, for dynamical studies like the longitudinal momentum spectrum of created particles, one has to solve the underlying Dirac equation for the fermionic system (the Klein-Gordon equation for a scalar field) interacting with the external electromagnetic field [15-22]. In order to have an analytically tractable dynamical description for the system of an unstable vacuum in the presence of the external field, two broad classes of theoretical formulations based on the spatiotemporal inhomogeneity of the external field were developed [23]. These are termed as the kinetic equation in the Wigner [24-26] and in the quasiparticle [27-29] representations. For a one-dimensional, spatially uniform, time-dependent electric field, the equivalence between these representations of the kinetic equation was shown in Ref. [30]. We use here the quasiparticle representation of the quantum kinetic equation (QKE) formalism [27,28,31-34] for the evolution of the quasiparticle vacuum in the presence of a time-dependent electric field in the mean field approximation, wherein the collisional effects of the created particles and backreaction force on the external electric field are neglected.

The production of particle-antiparticle pairs from the vacuum fluctuation in a time-dependent electric field $E(t)$ was seen as a field-induced phase transition (FIPT) via the $t$-noninvariant vacuum state because of the non-stationary Hamiltonian [29]. Here, the spontaneous symmetry breaking of the vacuum state takes place under time inversion, and consequently electron-positron pairs are generated which are the massive analogue of Goldstone bosons [35]. In order to quantify this symmetry breaking, one defines a complex order parameter $\Phi(\bar{p}, t)=2\left\langle 0_{\text {in }}\left|B_{\bar{p}}^{\dagger}(t) D_{-\bar{p}}^{\dagger}(t)\right| 0_{\text {in }}\right\rangle=$ $|\Phi(\bar{p}, t)| \exp (i \psi(\bar{p}, t))[29,36]$, where $B_{\bar{p}}^{\dagger}(t)$ and $D_{-\bar{p}}^{\dagger}(t)$ are the creation operators of particles and antiparticles with momentum $\pm \bar{p}$, respectively, in the quasiparticle representation in the time-dependent adiabatic particle number basis. FIPT was studied for the single and multisheeted electric pulses $[29,36]$. It was shown that the evolution of the modulus of order parameter $|\Phi(\bar{p}, t)|$ brings out three distinct stages or phases: namely, the quasielectron-positron plasma (QEPP) stage, the transient stage, and the final residual electron-positron plasma (REPP) stage. Here the QEPP stage is defined as the region where the smooth evolution of the modulus of the order parameter is defined by the quasienergy $\omega(\bar{p}, t)=\sqrt{m^{2}+\bar{p}_{\perp}^{2}+P_{3}^{2}(t)}$, where the longitudinal quasimomentum $P_{3}(t)=p_{3}-e A(t)$. The REPP stage is defined as the region where the evolution of $|\Phi(t)|$ does not show any oscillation, and it reaches a constant value. The most intriguing stage is the transient stage, where a highly oscillating region separates the smoothly oscillating QEPP region and the REPP stage. The effect of subcycle field oscillations on these stages was also studied for different longitudinal momentum values $[36,37]$. However, the evolution of the phase of the complex order parameter $\psi(\bar{p}, t)$, to the best of our knowledge, has not been studied so far.

In this article, we study the evolution of the modulus and the phase of $\Phi(\bar{p}, t)$ and analyze their interrelation. Frequency chirp is an essential and integral part of ultrashort laser pulses. Frequency chirping changes the time period of subcycle oscillations of the external electric field, which further induces complexity in the evolution of the order parameter. We analyze this complexity as a function of linear and quadratic frequency chirp parameters.

We use the natural units where $\hbar=c=1$, and the corresponding Compton length and timescales are denoted as $\lambda_{e}=\tau_{e}=1 / m_{e}$. The critical electric field strength of QED is then given by $E_{S}=m_{e}^{2} / e$. Furthermore, the mass and the charge of the electron are taken to be unity. The rest of the article is organized as follows: In Sec. II, we describe the basic equations governing the evolution of the modulus and the phase of $\Phi(\bar{p}, t)$. Results are discussed in Sec. III. The article is concluded in Sec. IV.

\section{THEORY}

The kinetic equation used in this work has been derived using different techniques by various researchers $[28,32,33]$. Here we give the essential steps, closely following the derivation given $[28,34]$. The basic formulation of fermionic pair production can be derived from the Dirac equation for the matter field $\Psi(x)$ :

$$
\left(i \gamma^{\mu} \partial_{\mu}-e \gamma^{\mu} A_{\mu}-m\right) \Psi(x)=0 .
$$

Here, we have assumed that the external electric field is a classical background, and we take a one-dimensional spatially uniform but time-dependent electric field which is characterized by the four-vector potential $A_{\mu}=(0,0,0, A(t)) \cdot \gamma$ matrices are the usual gamma matrices for the Dirac spinor field. The electric field can easily be calculated by $E(t)=-d A(t) / d t$. With the above mentioned gauge field, we take the eigenstates of the Dirac equation as

$$
\psi_{\bar{p}, r}^{( \pm)}(x)=\left[i \gamma^{0} \partial_{0}+\gamma^{k} p_{k}-e \gamma^{3} A(t)+m\right] \chi^{( \pm)}(\bar{p}, t) R_{r} e^{i \bar{p} \cdot \bar{x}},
$$


where $k=1,2,3$, and the superscript $( \pm)$ denotes eigenstates with positive and negative energies. $R_{r}$ is the eigenvector of the matrix $\gamma^{0} \gamma^{3}$, and the functions $\chi^{( \pm)}(\bar{p}, t)$ satisfy the oscillator-type equation [28]

$$
\ddot{\chi}^{( \pm)}(\bar{p}, t)=-\left(\omega^{2}(\bar{p}, t)+i e \dot{A}(t)\right) \chi^{( \pm)}(\bar{p}, t) .
$$

Now, one can construct the massive field operator $\Psi(x)$ with the eigenstates as

$$
\Psi(x)=\sum_{r, \bar{p}}\left(\psi_{\bar{p}, r}^{(-)}(x) b_{\bar{p}, r}\left(t_{0}\right)+\psi_{\bar{p}, r}^{(+)}(x) d_{-\bar{p}, r}^{\dagger}\left(t_{0}\right)\right),
$$

where $b_{\bar{p}, r}\left(t_{0}\right), b_{\bar{p}, r}^{\dagger}\left(t_{0}\right)$ and $d_{\bar{p}, r}\left(t_{0}\right), d_{\bar{p}, r}^{\dagger}\left(t_{0}\right)$ are the annihilation and creation operators for the particle and antiparticle with momentum $\bar{p}$ and spin $r$ in the in-state $\left|0_{\text {in }}\right\rangle$ at $t=t_{0}$, and they satisfy the anticommutation relation

$$
\left\{b_{\bar{p}, r}\left(t_{0}\right), b_{\bar{p}, r}^{\dagger}\left(t_{0}\right)\right\}=\left\{d_{\bar{p}, r}\left(t_{0}\right), d_{\bar{p}, r}^{\dagger}\left(t_{0}\right)\right\}=\delta_{r r^{\prime}} \delta_{\bar{p} \bar{p}^{\prime}} .
$$

Now the evolution in the presence of the time-dependent field affects the vacuum state and mixes state with positive and negative energies, which induces nondiagonal terms in the Hamiltonian. The diagonalization is done by the timedependent Bogoliubov transformation:

$$
\begin{aligned}
& b_{\bar{p}, r}(t)=\alpha_{\bar{p}}(t) b_{\bar{p}, r}\left(t_{0}\right)+\beta_{\bar{p}}(t) d_{-\bar{p}, r}^{\dagger}\left(t_{0}\right), \\
& d_{\bar{p}, r}(t)=\alpha_{-\bar{p}}(t) d_{\bar{p}, r}\left(t_{0}\right)-\beta_{-\bar{p}}(t) b_{-\bar{p}, r}^{\dagger}\left(t_{0}\right),
\end{aligned}
$$

with $\left|\alpha_{\bar{p}}(t)\right|^{2}+\left|\beta_{\bar{p}}(t)\right|^{2}=1$. The Bogoluibov coefficients $\alpha_{\bar{p}}(t), \beta_{\bar{p}}(t)$ satisfy the coupled equations

$$
\begin{gathered}
\dot{\alpha}_{\bar{p}}(t)=\frac{e E(t) \epsilon_{\perp}}{2 \omega^{2}(\bar{p}, t)} \beta_{\bar{p}}^{*}(t) e^{2 i \Theta(\bar{p}, t)}, \\
\dot{\beta}_{\bar{p}}^{*}(t)=-\frac{e E(t) \epsilon_{\perp}}{2 \omega^{2}(\bar{p}, t)} \alpha_{\bar{p}}(t) e^{-2 i \Theta(\bar{p}, t)},
\end{gathered}
$$

where $\epsilon_{\perp}=\sqrt{m^{2}+p_{\perp}^{2}}$ is the transverse energy, $e$ is the electric charge, and $\Theta(\bar{p}, t)=\int_{t_{0}}^{t} d t^{\prime} \omega\left(\bar{p}, t^{\prime}\right)$ gives the dynamical phases accumulated between the initial and final states.

Here one can define the creation and annihilation operators of particles antiparticles in adiabatic particle number basis by absorbing the fast oscillation as

$$
B_{\bar{p}, r}(t)=b_{\bar{p}, r}(t) e^{-i \Theta(\bar{p}, t)}, \quad D_{\bar{p}, r}(t)=d_{\bar{p}, r}(t) e^{-i \Theta(\bar{p}, t)}
$$

The operators $B_{\bar{p}, r}(t)$ and $D_{\bar{p}, r}(t)$ satisfy the Heisenberglike equations of motion

$$
\begin{aligned}
& \frac{d B_{\bar{p}, r}(t)}{d t}=-\frac{e E(t) \epsilon_{\perp}}{2 \omega^{2}(\bar{p}, t)} D_{-\bar{p}, r}^{\dagger}(t)+i\left[H(t), B_{\bar{p}, r}(t)\right], \\
& \frac{d D_{\bar{p}, r}(t)}{d t}=\frac{e E(t) \epsilon_{\perp}}{2 \omega^{2}(\bar{p}, t)} B_{-\bar{p}, r}^{\dagger}(t)+i\left[H(t), D_{\bar{p}, r}(t)\right],
\end{aligned}
$$

where the quasiparticle Hamiltonian $H(t)$ is given by

$$
H(t)=\sum_{r, \bar{p}} \omega(\bar{p}, t)\left(B_{\bar{p}, s}^{\dagger}(t) B_{\bar{p}, r}(t)-D_{-\bar{p}, r}(t) D_{-\bar{p}, r}^{\dagger}(t)\right) .
$$

We now define the occupation number of the electron in the time-dependent basis with spin $r$ and momentum $\bar{p}$ for the initial vacuum state: $f_{r}(\bar{p}, t)=\left\langle 0_{\text {in }}\left|B_{\bar{p} r}^{\dagger}(t) B_{\bar{p} s}(t)\right| 0_{\text {in }}\right\rangle$. Similarly, one can also define the occupation number of the positron: $\bar{f}_{r}(-\bar{p}, t)=\left\langle 0_{\text {in }}\left|D_{-\bar{p} s}^{\dagger}(t) D_{-\bar{p}, r}(t)\right| 0_{\text {in }}\right\rangle$. Here $f_{r}(\bar{p}, t)=\bar{f}_{r}(-\bar{p}, t)$ due to the charge conjugation invariance. Now, $f_{r}(\bar{p}, t)$ and $\bar{f}_{r}(-\bar{p}, t)$ will serve as single-particle distribution functions in the quasiparticle representation $[28,29]$. The evolution of $f_{r}(\bar{p}, t)$ satisfies the equation

$$
\frac{d f_{r}(\bar{p}, t)}{d t}=-\frac{e E(t) \epsilon_{\perp}}{2 \omega^{2}(\bar{p}, t)} \operatorname{Re}\left\{\Phi_{r}(\bar{p}, t)\right\}
$$

where $\Phi_{r}(\bar{p}, t)=\left\langle 0_{\text {in }}\left|D_{-\bar{p} r}(t) B_{\bar{p} r}(t)\right| 0_{\text {in }}\right\rangle$ is the particleantiparticle correlation function, which satisfies the evolution equation

$$
\frac{d \Phi_{r}(\bar{p}, t)}{d t}=\frac{e E(t) \epsilon_{\perp}}{\omega^{2}(\bar{p}, t)}\left[2 f_{r}(\bar{p}, t)-1\right]-2 i \omega(\bar{p}, t) \Phi_{r}(\bar{p}, t),
$$

provided that $f_{r}(\bar{p}, t)=\bar{f}_{r}(-\bar{p}, t)$ is used. In general, $\Phi_{r}(\bar{p}, t)=u_{r}(\bar{p}, t)+i v_{r}(\bar{p}, t)$, which turns into the above equation:

$$
\begin{aligned}
& \frac{d u_{r}(\bar{p}, t)}{d t}=\frac{e E(t) \epsilon_{\perp}}{\omega^{2}(\bar{p}, t)}\left[2 f_{r}(\bar{p}, t)-1\right]+2 \omega(\bar{p}, t) v_{r}(\bar{p}, t) \\
& \frac{d v_{r}(\bar{p}, t)}{d t}=-2 \omega(\bar{p}, t) u_{r}(\bar{p}, t) .
\end{aligned}
$$

In particular, we have a set of three differential equations for the complete dynamical evolution of the vacuum electron-positron pair production process, which are listed below. [We have absorbed the minus sign which appears in the above equations for $f(\bar{p}, t), u(\bar{p}, t)$, and $v(\bar{p}, t)$ into the definition of $u(\bar{p}, t)$; i.e., $u(\bar{p}, t) \rightarrow-u(\bar{p}, t)$.] 


$$
\begin{aligned}
& \frac{d f(\bar{p}, t)}{d t}=\frac{e E(t) \epsilon_{\perp}}{2 \omega^{2}(\bar{p}, t)} u(\bar{p}, t), \\
& \frac{d u(\bar{p}, t)}{d t}=\frac{e E(t) \epsilon_{\perp}}{\omega^{2}(\bar{p}, t)}[1-2 f(\bar{p}, t)]-2 \omega(\bar{p}, t) v(\bar{p}, t), \\
& \frac{d v(\bar{p}, t)}{d t}=2 \omega(\bar{p}, t) u(\bar{p}, t) .
\end{aligned}
$$

The anomalous average term $u(\bar{p}, t)$ which represents vacuum polarization effects plays an important role in the source term of the pair production. In fact, this term gives information about the quantum statistic character via $[1-2 f(\bar{p}, t)]$, due to the Pauli exclusion principle. The term $v(\bar{p}, t)$ denotes some kind of countering term to the pair production, which is basically the pair annihilation in the vacuum excitation process. One can also combine the above set of first-order differential equation into a single first-order integrodifferential equation

$$
\begin{aligned}
\frac{d f(\bar{p}, t)}{d t}= & \frac{e E(t) \epsilon_{\perp}^{2}}{2 \omega^{2}(\bar{p}, t)} \int_{-\infty}^{t} d t^{\prime} \frac{e E\left(t^{\prime}\right)}{\omega^{2}\left(\bar{p}, t^{\prime}\right)} \\
& \times\left[1-2 f\left(\bar{p}, t^{\prime}\right)\right] \cos \left[2 \Theta\left(\bar{p} ; t, t^{\prime}\right)\right],
\end{aligned}
$$

which will serve as the basis of the quantum transport equation of the vacuum particle pair production [27,31], where the non-Markovian character or the memory effect is present via the terms $[1-2 f(\bar{p}, t)]$ and the highly oscillating kernel $\cos \left[2 \Theta\left(\bar{p} ; t, t^{\prime}\right)\right]$.

Using the first integral of motion, $(1-2 f(\bar{p}, t))^{2}+$ $|\Phi(\bar{p}, t)|^{2}=1$, we get the following nonlinear coupled differential equations for the evolution of the modulus $|\Phi(\bar{p}, t)|$ and the phase $\psi(\bar{p}, t)$ of the order parameter:

$$
\begin{aligned}
\frac{d|\Phi(\bar{p}, t)|}{d t} & =\frac{e E(t) \epsilon_{\perp}}{\omega^{2}(\bar{p}, t)} \cos \psi(\bar{p}, t) \sqrt{1-|\Phi(\bar{p}, t)|^{2}}, \\
\frac{d \psi(\bar{p}, t)}{d t} & =2 \omega(\bar{p}, t)-\frac{e E(t) \epsilon_{\perp}}{\omega^{2}(\bar{p}, t)} \sin \psi(\bar{p}, t) \frac{\sqrt{1-|\Phi(\bar{p}, t)|^{2}}}{|\Phi(\bar{p}, t)|} .
\end{aligned}
$$

The particle acceleration is governed by $d P_{3}(t) / d t=$ $e E(t)$ in the presence of the time-dependent electric field $E(t)$.

The electric field in this study is taken as the multisheeted Sauter pulse, which is considered to describe well the resultant field of counterpropagating ultrashort laser pulses in the focal region:

$$
E(t)=E_{0} \cosh ^{-2}(t / \tau) \cos \left(\alpha t^{3}+\beta t^{2}+\omega_{0} t\right),
$$

where $\beta$ and $\alpha$ are the linear and quadratic frequency chirp parameters, respectively; $\omega_{0}$ is the central frequency of the laser electric field oscillation, with $\tau$ being the total pulse length. A single-sheeted Sauter pulse corresponds to $\alpha=\beta=\omega_{0}=0$. In the present study, we have taken a laser radiation field with frequency $25 \mathrm{keV}$, which falls in the XFEL [38] region. However, the intensity $I=1.15 \times 10^{27} \mathrm{~W} / \mathrm{cm}^{2}$, which we consider here, is not attainable in the XFELs presently or even in the foreseeable future.

\section{RESULTS}

We solve Eq. (16) numerically for the evolution of $|\Phi(\bar{p}, t)|$ and $\psi(\bar{p}, t)$ with the initial condition $|\Phi(\bar{p}, t \rightarrow-\infty)|=0$. The phase $\psi(\bar{p}, t)$ is defined only up to an arbitrary additive constant. For definiteness, we begin at $t=-10 \tau$ with $|\Phi(\bar{p}, t=-10 \tau)|=10^{-16}$ and $\psi(\bar{p}, t=-10 \tau)=\pi / 4$, so as to have $u=v=10^{-16} / \sqrt{2}$ initially. Here we have used the ordinary differential equation solver ODE113 (a built-in package for solving nonstiff differential equations with variable step and variable order) in MATLAB [39] to solve Eq. (16). As we are dealing with very small numbers, we have taken care about numerical errors. The relative and absolute error tolerances are set to $10^{-14}$ and $10^{-15}$, respectively for solving Eq. (16).

As mentioned before, the complete evolution of the modulus of the order parameter $|\Phi(\bar{p}, t)|$ was shown to go through three distinct stages: namely, the initial QEPP stage, the transient stage, and the final REPP stage of the created electron-positron pairs by the external time-dependent electric field of single-sheeted Sauter pulses and multisheeted Gaussian pulses [36,37]. However, it is not clear what is the origin of the transient stage. Furthermore, the evolution of the phase $\psi(\bar{p}, t)$ has not been studied so far. We therefore revisit these cases. $|\Phi(\bar{p}, t)|$ and $\psi(\bar{p}, t)$ are plotted as a function of time for single and multisheeted Sauter pulses without any frequency chirp $\left(\omega_{0} \tau=5\right.$, $\alpha=\beta=0)$ in Fig. 1. It is seen in Fig. 1(a) that $|\Phi(\bar{p}, t)|$ increases monotonically with time in the QEPP region for the single-sheeted Sauter pulse up to $t=0$, at which point the electric field reaches its maximum value, and thereafter $|\Phi(\bar{p}, t)|$ decreases. $|\Phi(\bar{p}, t)|$, after decreasing to a certain value, shows rapid oscillations and thus undergoes transition from QEPP to a transient region before settling down to the REPP state as a consequence of FIPT wherein $|\Phi(\bar{p}, t)|$ reaches a constant value different from zero. The phase $\psi(\bar{p}, t)$ remains almost constant before increasing rapidly about the time the transient stage in the evolution of $|\Phi(\bar{p}, t)|$ appears; see Fig. 1(c). For the multisheeted Sauter pulse, the evolution of $|\Phi(\bar{p}, t)|$ in Fig. 1(b) shows periodic oscillations corresponding to the subcycle structure of the electric field in the QEPP region. The transient region appears later and becomes elongated before it reaches the final REPP state. $\psi(\bar{p}, t)$ is found to increase slowly in the QEPP region and then rapidly at the onset of the transient stage; note the abrupt change of slope in the linear growth of $\psi(\bar{p}, t)$ in Fig. 1(d). The blowup of the evolution of $\psi(\bar{p}, t)$ in the transient stage shows a staircase-like structure. 

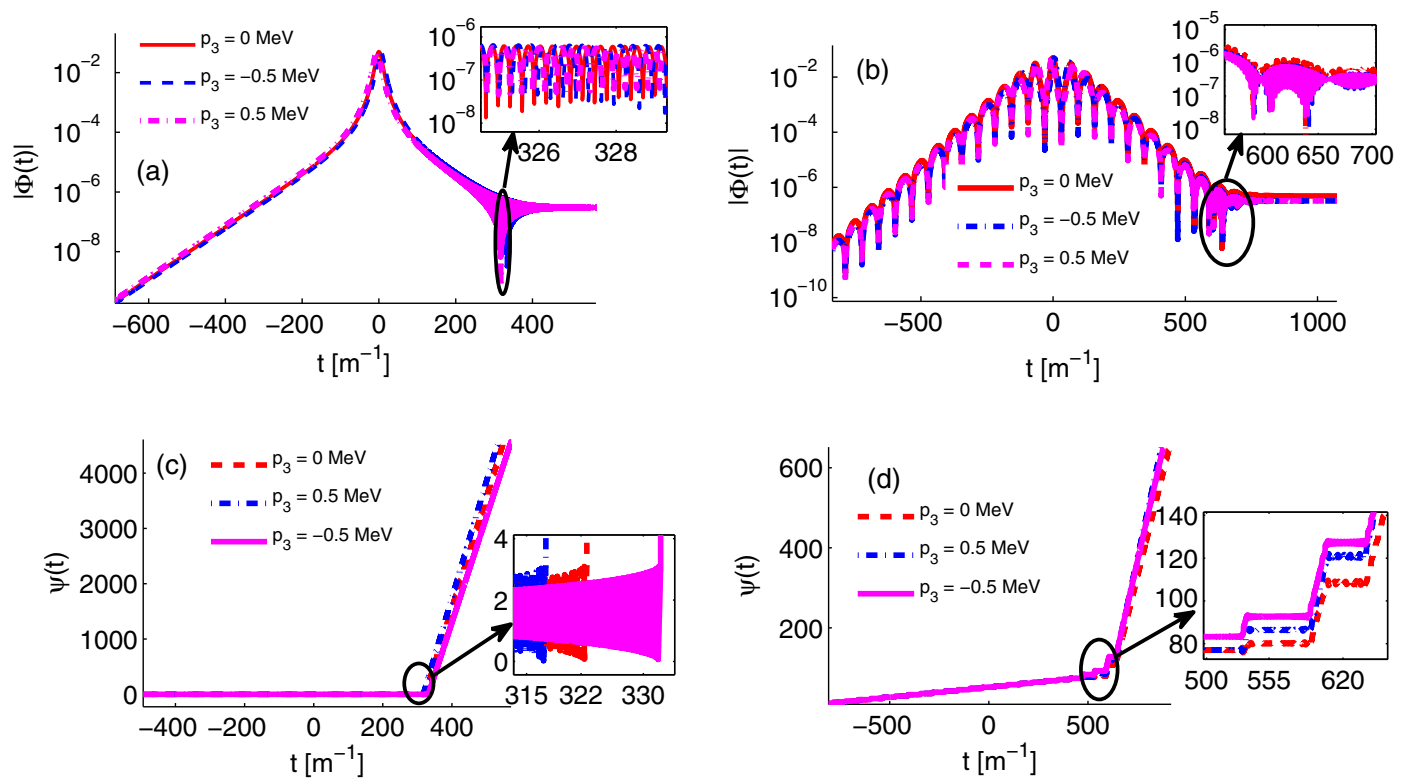

FIG. 1. Evolution of the modulus $|\Phi(\bar{p}, t)|$ and the phase $\psi(\bar{p}, t)$ of the order parameter: (a),(c) for a single-sheeted Sauter pulse; (b),(d) for a multisheeted $\left(\omega_{0} \tau=5\right)$ Sauter pulse without frequency chirping $(\alpha=\beta=0)$ for the longitudinal momentum values $p_{3}=0 \mathrm{MeV}, \mp 0.5 \mathrm{MeV}$. All the measurements are taken in electron mass units, and the transverse momentum $\bar{p}_{\perp}=0$. The field parameters are $E_{0}=0.1$ and $\tau=100$. The insets show a magnified view of the evolution in the transient region.

As seen in Eq. (16), the evolution of $|\Phi(\bar{p}, t)|$ is governed by the temporal profiles of the electric field $E(t)$ and the corresponding vector potential $A(t)$ through the ratio $E(t) / \omega^{2}(\bar{p}, t)$ (note that $\epsilon_{\perp}=e=1$ ) and also by the phase term $\cos \psi(\bar{p}, t)$. As $\psi(\bar{p}, t)$ remains nearly constant, the QEPP stage is largely controlled by $E(t) / \omega^{2}(\bar{p}, t)$, as seen in Fig. 1(a). For the single-sheeted pulse as shown in Fig. 2(a), the electric field profile is smooth, having its maximum at $t=0$, while the vector potential is large in magnitude on either side of the electric field maximum, resulting in a sharper temporal profile of $E(t) / \omega^{2}(\bar{p}, t)$. In Refs. [36,37,40], the temporal profile of $|\Phi(\bar{p}, t)|$ in the QEPP stage was compared to that of $|E(t)|$. However, the much sharper profile of $|\Phi(\bar{p}, t)|$, particularly near the center of the pulse, and the faster decay thereof in the tail regions is better explained by $|E(t)| / \omega^{2}(\bar{p}, t)$ than
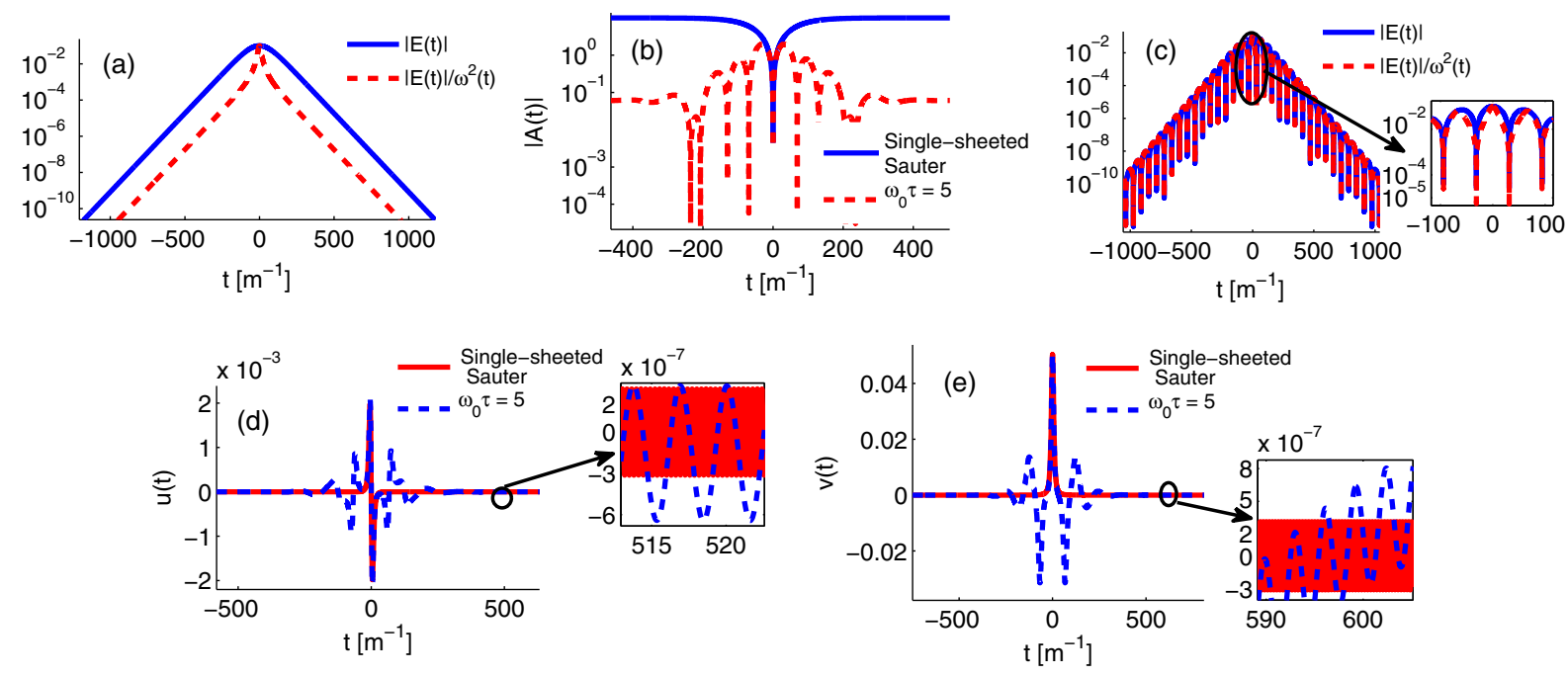

FIG. 2. (a),(c) $E(t),|E(t)| / \omega^{2}(\bar{p}, t)$ for single-sheeted and multisheeted $\left(\omega_{0} \tau=5\right)$ Sauter pulses, respectively. (b) $A(t)$ for single and multisheeted pulses. (d),(e) Evolutions of $u(\bar{p}, t)$ and $v(\bar{p}, t)$, respectively, for single and multisheeted pulses, with insets showing a magnified view of the evolution in the transient stage. All the measurements are taken in electron mass units. The field parameters are $E_{0}=0.1, \tau=100$, the central frequency of the pulse is $\omega_{0}=0.05$, and the linear and quadratic frequency chirp parameters are $\beta=\alpha=0$. Transverse and longitudinal momenta are taken to be zero. 

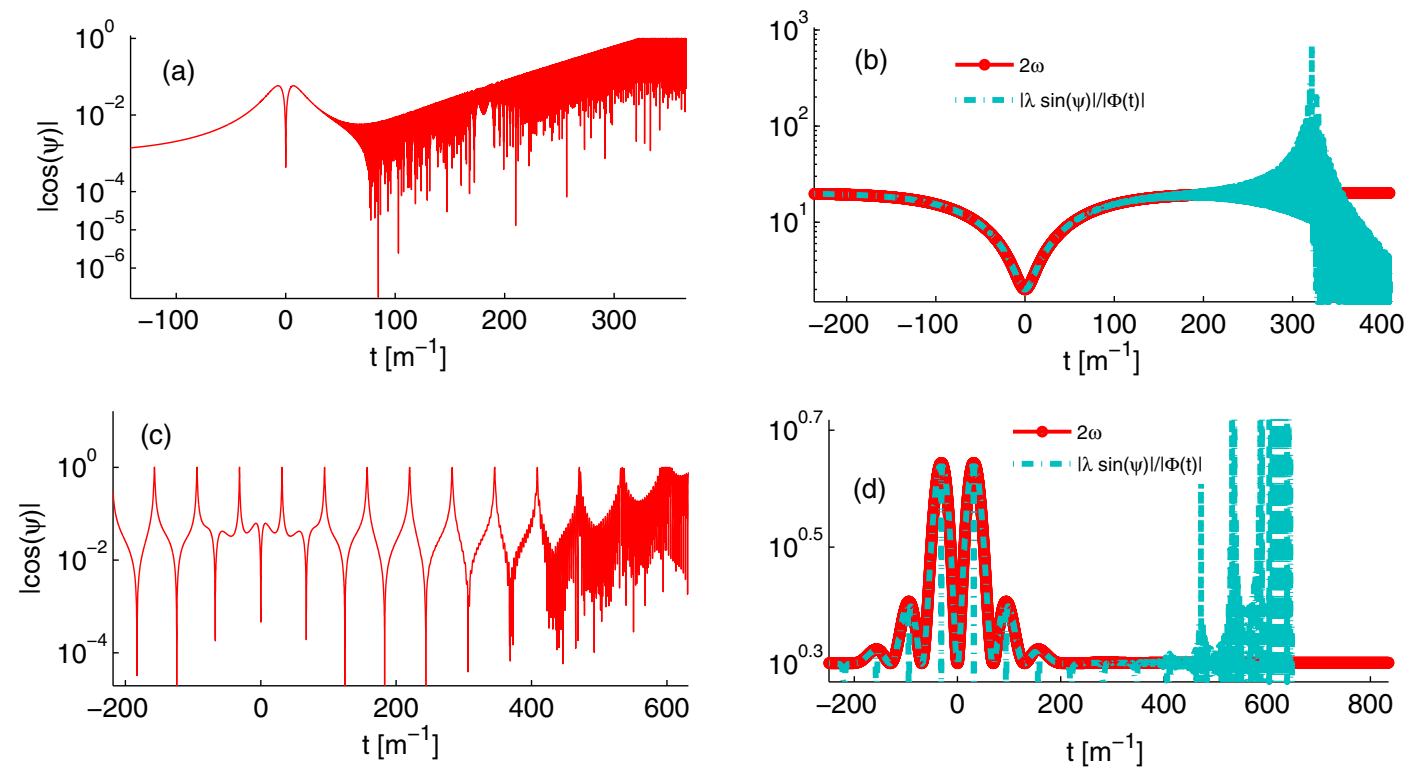

FIG. 3. (a),(c) $|\cos \psi(\bar{p}, t)|$ for single-sheeted and multisheeted Sauter pulses, respectively. (b), (d) $2 \omega(\bar{p}, t)$ and $|\lambda \sin \psi(\bar{p}, t)| /$ $|\Phi(\bar{p}, t)|$ for single-sheeted and multisheeted Sauter pulses, respectively. Here, $\lambda=E(t) / \omega^{2}(\bar{p}, t)$. The longitudinal and transverse momenta are $p_{3}=p_{\perp}=0$. The field parameters are $E_{0}=0.1, \tau=100$.

$|E(t)|$; see Fig. 2(a). The formation of the transient region takes place because of the sudden rapid increase in the value of the phase $\psi(\bar{p}, t)$, which makes $\cos \psi(\bar{p}, t)$ [on the right-hand side of Eq. (16)] and hence $|\Phi(\bar{p}, t)|$ oscillate rapidly; see Fig. 3(a). Once the electric field gets vanishingly small, $d|\Phi(\bar{p}, t)| / d t=0$, and we have a constant value of $|\Phi(\bar{p}, t)|$ in the REPP region. In this region $d \psi(\bar{p}, t) / d t=2 \omega(\bar{p}, t)$, and hence $\Phi(\bar{p}, t) \sim \Phi_{R} e^{2 i \omega t}$, where $\Phi_{R}$ is the constant value of $\Phi(\bar{p}, t)$. $\Phi_{R}$ being largest for $p_{3}=0$ and the same for $p_{3}= \pm 0.5 \mathrm{MeV}$ is consistent with the well-known fact that the asymptotic momentum spectrum of pairs created by the single-sheeted pulse is centered at $p_{3}=0$ and is symmetric about it [41]. At this point, it is worthwhile noting that in the QEPP stage for $t \geq 0$ and in the transient stage, $|\Phi(\bar{p}, t)|$ is largest for $p_{3}=-0.5 \mathrm{MeV}$, which suggests that the (quasi)particle momentum spectrum should be centered about a negative value of $p_{3}$. As reported in a recent work [42], this indeed is the case. For the multisheeted pulse, as shown in Fig. 2(c), the electric field oscillates within the smooth envelope. In contrast to the single-sheeted field case, the vector potential is much suppressed in the tail region of the field, having oscillatory structure in the center; see Fig. 2(b). The resulting $E(t) / \omega^{2}(\bar{p}, t)$ has a temporal profile close to that of $E(t)$, except near the pulse center. The QEPP region is consequently broader, and the temporal profile of $|\Phi(\bar{p}, t)|$ in this region is modified by the subcycle structures of the electric field. In this case too, the temporal profile of $|\Phi(\bar{p}, t)|$ in the QEPP stage is well explained by that of $|E(t)| / \omega^{2}(\bar{p}, t)$. The rapid oscillation of $|\Phi(\bar{p}, t)|$ in the transient region in this case is governed by the oscillations in $E(t), \cos \psi(\bar{p}, t)$, and $A(t)$; therefore the transient region is elongated and the modulation effect is seen in Fig. 1(b). In the QEPP region, the counterterm $v(\bar{p}, t)$ governing the depolarization and pair annihilation is stronger than $u(\bar{p}, t)$, which is responsible for the polarization and pair creation [Figs. 2(d)-(e)]. Both $u(\bar{p}, t)$ and $v(\bar{p}, t)$ oscillate with varying amplitudes which are large in the center of the pulse. The decrease in the amplitude of $v(\bar{p}, t)$ in moving away from the center is more than that of $u(\bar{p}, t)$. In the transient region, both the amplitudes are nearly the same, before becoming identical in the REPP stage.

\section{A. Effect of frequency chirping on field-induced phase transition}

It is clear from the results discussed so far that the complexity in the evolution of the modulus and the phase of the order parameter, particularly in the transient stage, is because of the nonlinear coupling in the dynamical equations governing the evolution of the modulus and the phase. As demonstrated above, in the QEPP region, the evolution of the modulus is mostly governed by both the electric field $E(t)$ and the vector potential $A(t)$. The evolution of the phase, on the other hand, is governed by two distinct terms which contain all the dynamic variables. In the QEPP stage, where the phase evolves slowly and smoothly, the two terms seem to balance each other. The transient stage arises when this dynamic balance is lost and hence there is steep increase in the phase over a very small duration. After the transient stage, the dynamics of the phase and modulus of the order parameter are decoupled; see Fig. 3.

The presence of frequency chirping in effect makes frequency time dependent. This, in turn, affects the number 

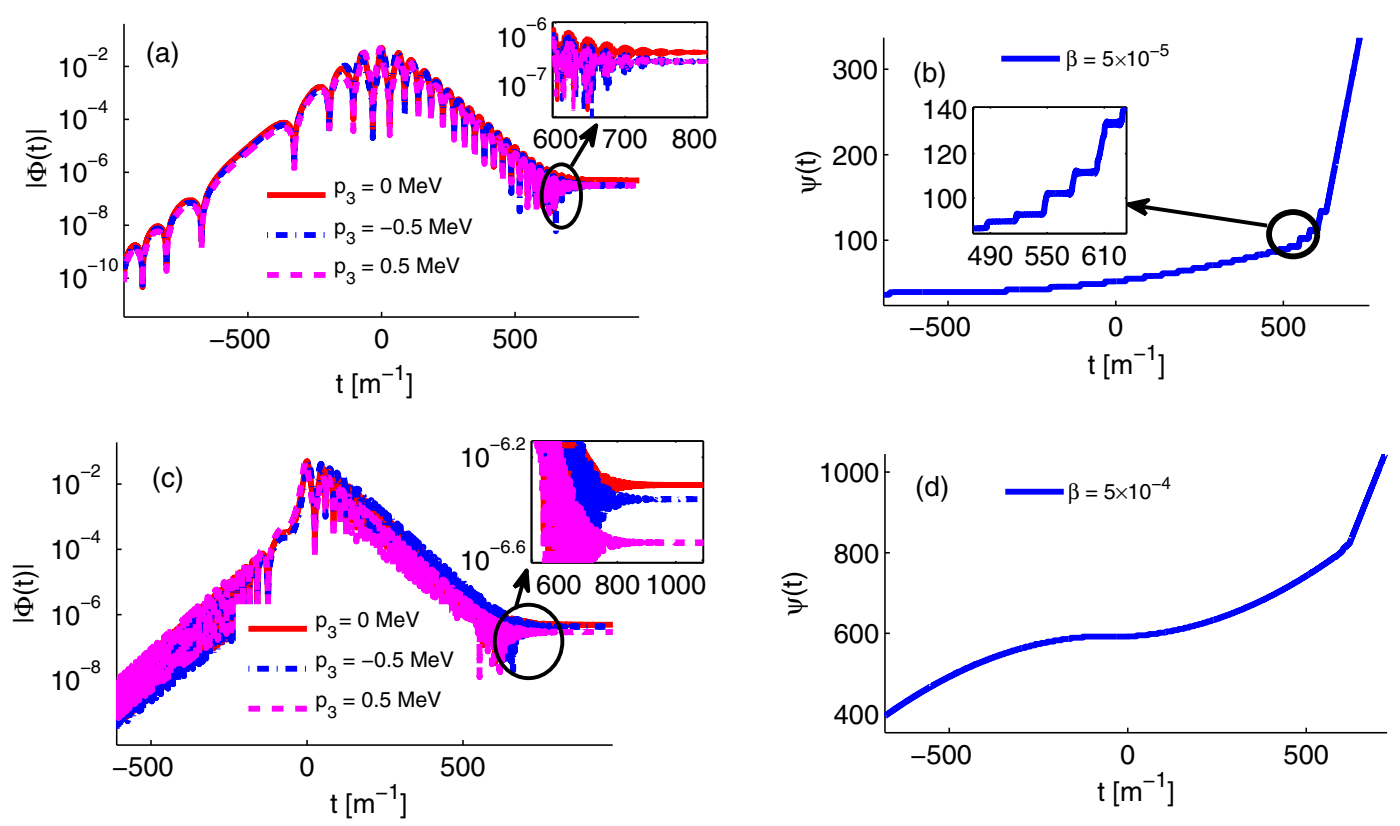

FIG. 4. Evolution of $|\Phi(\bar{p}, t)|$ and $\psi(\bar{p}, t)$ for a time-dependent multisheeted Sauter pulse with linear frequency chirp. (a), (b) $\beta=5 \times 10^{-5}$. (c), (d) $\beta=5 \times 10^{-4}$. In (a) and (c), the longitudinal momentum $p_{3}=0, \pm 0.5 \mathrm{MeV}$. In (b) and (d), $p_{3}=0$. The transverse momentum $p_{\perp}=0$ for all the cases. $\omega_{0} \tau=5$. Other parameters are same as in Fig. 1 .

of subcycle oscillations within the envelope. In the presence of linear frequency chirp $\beta$, the subcycle oscillations are asymmetric about $t=0$. As we have taken the positive value of $\beta$, the number of oscillations within the pulse envelope is less for $t<0$ than for $t>0$. Hence, the evolution of $|\Phi(\bar{p}, t)|$ shows irregular oscillations in the QEPP state (Fig. 4). As $\beta$ is increased further, the oscillations become more rapid and are spread throughout the QEPP region, as seen in Fig. 4(c). The evolution can be understood qualitatively by looking at the temporal profiles of $E(t), A(t)$, and also the ratio $|E(t)| / \omega^{2}(\bar{p}, t)$, as shown in Fig. 5. For $\beta=5 \times 10^{-5}$, the effect of frequency chirping is small. Hence, the evolution of $|\Phi(\bar{p}, t)|$, as in the case of the multisheeted Sauter pulse discussed above, follows the electric field profile in the QEPP stage, and the transient stage is marked by the sudden change in the evolution of the phase. For $\beta=5 \times 10^{-4}$, however, there is much more asymmetry in the electric field profile, and the vector potential is large and constant for $t<0$, before undergoing quick oscillations near $t=0$ and attaining a constant value thereafter. The evolution of $|\Phi(\bar{p}, t)|$ with enhanced oscillation frequencies in the QEPP stage follows the temporal profile of $|E(t)| / \omega^{2}(\bar{p}, t)$.

We now study the effect of varying the linear chirp parameter $\beta$ in the presence of a fixed quadratic chirp $\left(\alpha=1 \times 10^{-6}\right)$ on FIPT. For small values of $\beta$, the inequality $\omega_{0}>\alpha \tau^{2}>\beta \tau$ holds, and the quadratic chirp dominates the evolution of $|\Phi(\bar{p}, t)|$. The evolution shows the formation of a pretransient stage in the QEPP region for values of $t<0$ (before the electric field reaches its maximum value $E_{0}$ ). This is shown in Fig. 6(a). The onset of the pretransient region, like in the transient stage, is marked by the dominance of the $\left(E(t) / \omega^{2}(\bar{p}, t)\right)(\sin \psi(\bar{p}, t) /|\Phi(\bar{p}, t)|)$ term over the $2 \omega(\bar{p}, t)$ term in controlling the dynamics of $\psi(\bar{p}, t)$ [Fig. 6(d)]. This causes rapid oscillations in $\cos \psi(\bar{p}, t)$ [Fig. 6(c)], and hence in $|\Phi(\bar{p}, t)|$ [Fig. 6(a)] around the temporal region near the pretransient stage. However, the pretransient stage gets suppressed for higher values of $\beta$ to
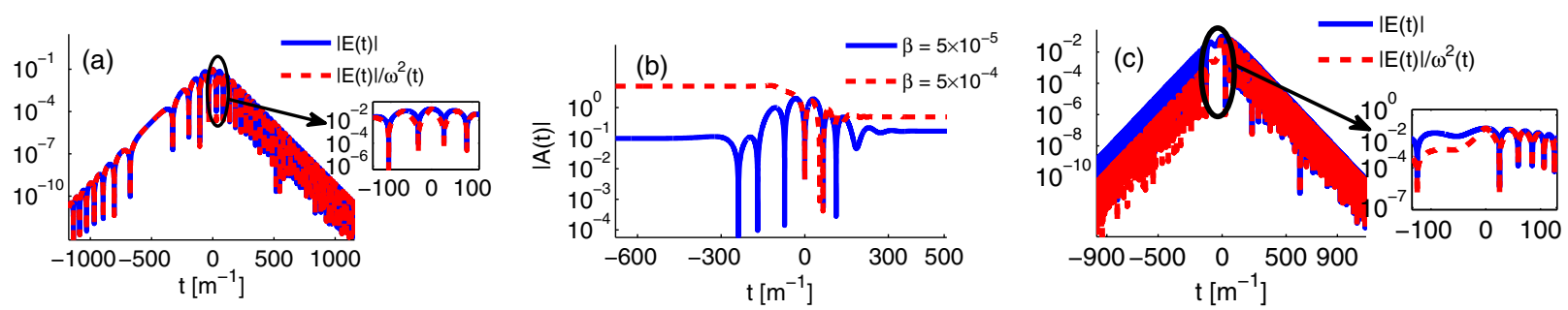

FIG. 5. (a),(c) $|E(t)|$ and $|E(t)| / \omega^{2}(\bar{p}, t)$ for the two Sauter pulses used in Fig. 4 with $\beta=5 \times 10^{-5}$ and $\beta=5 \times 10^{-4}$, respectively. (b) $|A(t)|$ for both the Sauter pulses. Other parameters are same as in Fig. 2. 

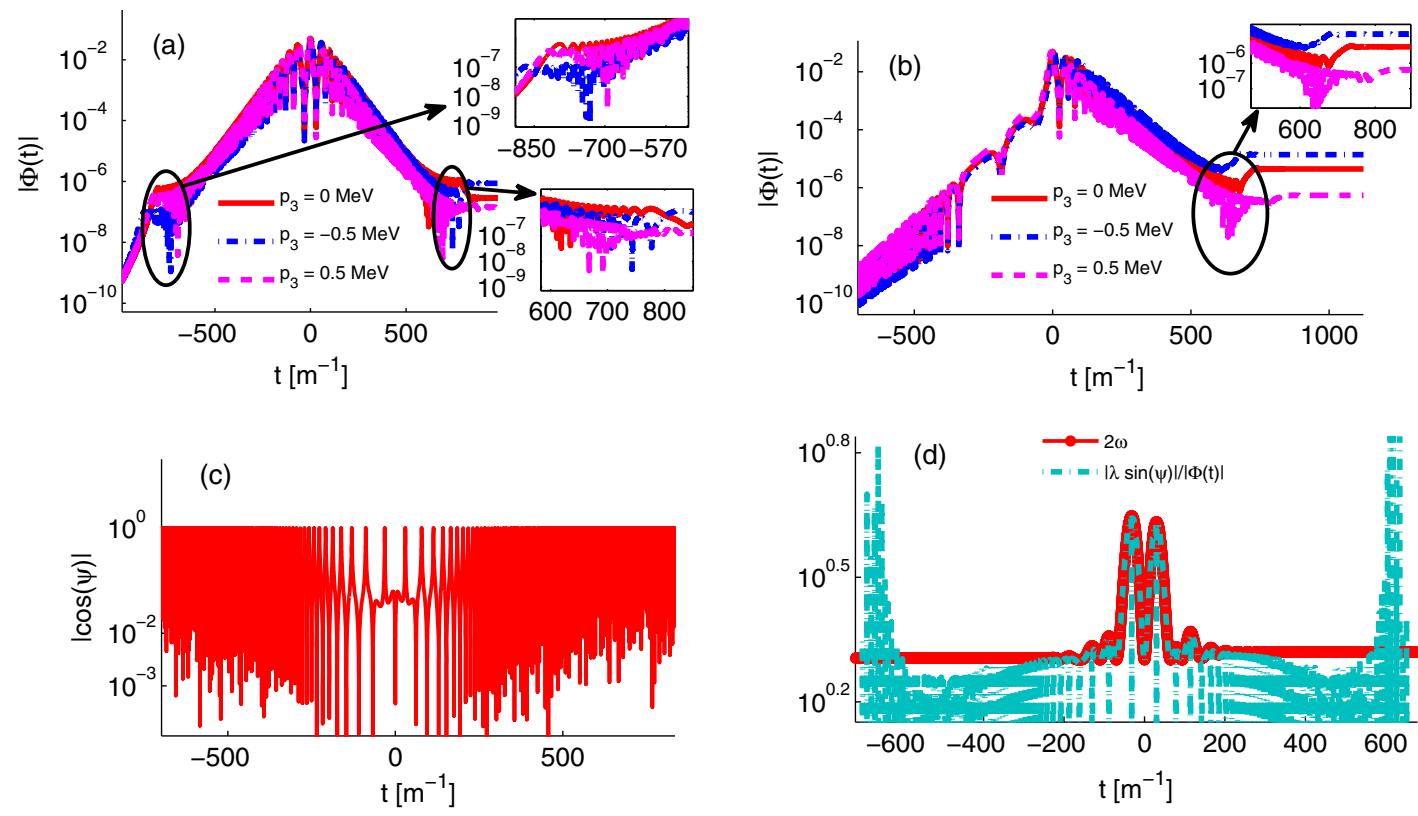

FIG. 6. (a) Evolution of $|\Phi(\bar{p}, t)|$ for a multisheeted Sauter pulse with linear chirp $\beta=5 \times 10^{-5}$, quadratic chirp $\alpha=1 \times 10^{-6}$, and the longitudinal momentum $p_{3}=0, \pm 0.5 \mathrm{MeV}$. (b) Same as in (a) for a Sauter pulse with a stronger linear frequency chirp, $\beta=5 \times 10^{-4}$. (c) $|\cos \psi(\bar{p}, t)|$, (d) $2 \omega(\bar{p}, t)$, and $|\lambda \sin \psi(\bar{p}, t)| /|\Phi(\bar{p}, t)|$ for the Sauter pulse with $\beta=5 \times 10^{-5}, \alpha=1 \times 10^{-6}$, and for the longitudinal momentum $p_{3}=0 . \omega_{0} \tau=5$. The transverse momentum $p_{\perp}=0$ for all cases. $\lambda=E(t) / \omega^{2}(\bar{p}, t)$. Other parameters are same as in Fig. 1.

give the uninterrupted QEPP region with irregular, fast, and spread out oscillations as seen in Fig. 6(b). It is seen that for higher values of $\beta$, the formation of the REPP stage takes place with a larger magnitude of the order parameter for the $p_{3}=-0.5 \mathrm{MeV}$ mode than for $p_{3}=0.5 \mathrm{MeV}$, suggesting
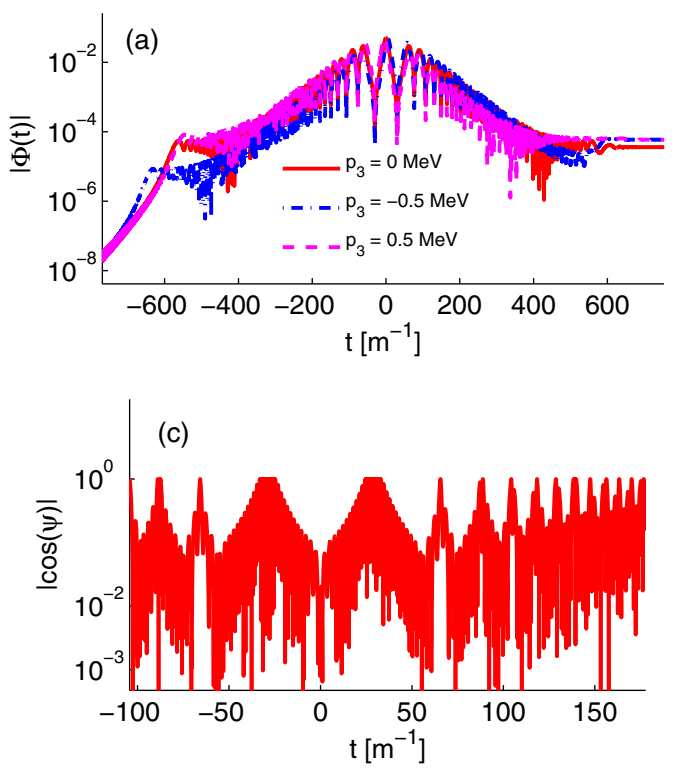

thereby that the momentum spectrum pairs created by the pulse are centered at a negative value of longitudinal momentum.

The effect of only quadratic chirping on FITP is shown in Fig. 7 for the longitudinal momentum values $p_{3}=0 \mathrm{MeV}$
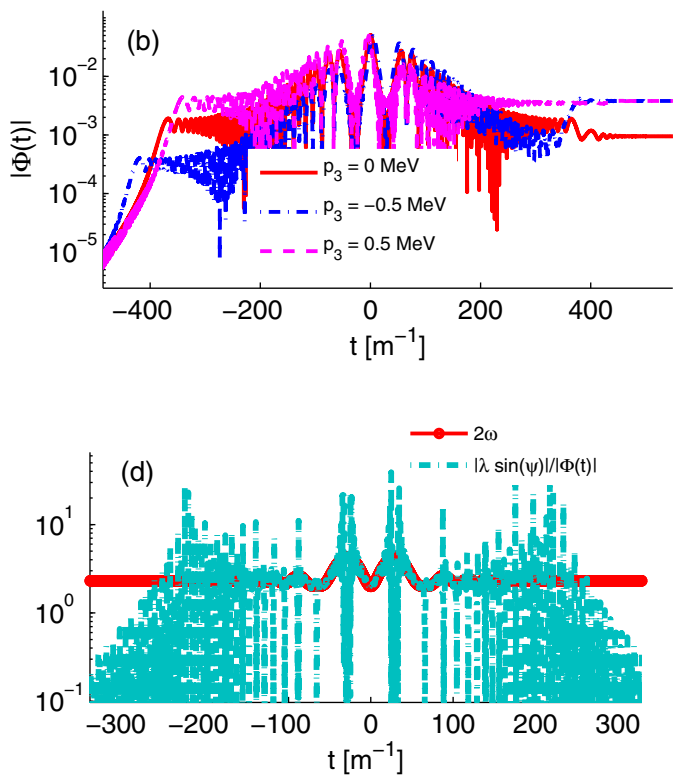

FIG. 7. (a) $|\Phi(\bar{p}, t)|$ for a multisheeted Sauter pulse with only quadratic chirp $(\beta=0)$ with $\alpha=2 \times 10^{-6}$. Longitudinal momentum $p_{3}=0, \pm 0.5 \mathrm{MeV}$. (b) Same as in (a) for $\alpha=5 \times 10^{-6}$. (c) $|\cos \psi(\bar{p}, t)|$, (d) $2 \omega(\bar{p}, t)$, and $|\lambda \sin \psi(\bar{p}, t)| /|\Phi(\bar{p}, t)|$ for $\beta=0$, $\alpha=5 \times 10^{-6}$, and $p_{3}=0$. The transverse momentum $p_{\perp}=0$ for all the cases. $\lambda=E(t) / \omega^{2}(\bar{p}, t)$. The other parameters are the same as in Fig. 1. 
and $p_{3}= \pm 0.5 \mathrm{MeV}$. For $\alpha=2 \times 10^{-6}$, the evolution of $|\Phi(\bar{p}, t)|$ shows high-frequency oscillations in the QEPP stage, as seen in Fig. 7(a). There is a formation of the pretransient region in this case. When the value of $\alpha$ is increased to $\alpha=5 \times 10^{-6}$, the pretransient and the transient regions move closer to the maximum of the electric field and hence get closer to each other. The higher the value of $\alpha$, the larger the shift. When the pretransient and transient stages are close enough, as in Fig. 7(b) for example, the rapid oscillations in $\cos \psi(\bar{p}, t)$ which set in at the pretransient stage continue till the transient stage; see Fig. 7(c). This should be compared with the evolution of $\cos \psi(\bar{p}, t)$ presented in Fig. 6(c), wherein the rapid oscillations of a relatively far-off pretransient stage do not extend all the way up to the transient stage. They are rather interrupted by slower oscillations in the central region of the pulse. An early occurrence of the transient stage leads to the formation of the REPP stage at earlier times as the value of $\alpha$ is increased. Consequently, the modulus of the order parameter in the REPP region increases with the increase in the value of $\alpha$. This results in the enhancement of the pair production rate. Furthermore, a clear separation for different momentum modes is seen in the pretransient and transient stages of evolution of the order parameter.

For quantitative estimation of the enhancement of the pair production rate due to the presence of quadratic chirp in the ultrashort laser pulse, we present the average number of created particles $n_{e^{+} e^{-}}=2 g \int d^{3} p /(2 \pi)^{3} f(\bar{p}, t \rightarrow \infty)$ for different values of the quadratic frequency chirp parameter $\alpha$ in Table I. Here, $g$ is the spin degeneracy factor. The average number of created particles increases by 5 orders of magnitude as the value of $\alpha$ is increased from $1 \times 10^{-6}$ to $6 \times 10^{-6}$. Such an enhancement in the pair production rate may be attributed to the increase in the subcycle oscillation frequency of the laser pulse away from the pulse center in the temporal envelope. The subcycle oscillation of the laser pulse may be described by an effective time-dependent frequency $\omega_{\text {eff }}(t)=\omega_{0}+\alpha t^{2}$. With the values of the subcycle oscillation frequency

TABLE I. The average number of created particles $n_{e^{+} e^{-}}$with different values of the quadratic frequency chirp parameter $\alpha$ for the multisheeted Sauter pulse with $\omega_{0} \tau=5$. Here $E_{0}=0.1$, $\tau=100$.

\begin{tabular}{lc}
\hline \hline$\alpha$ & $n_{e^{+} e^{-}}=4 \int \frac{d^{3} p}{(2 \pi)^{3}} f(\bar{p}, t=10 \tau)$ \\
\hline $1 \times 10^{-6}$ & $1.056 \times 10^{-10}$ \\
$2 \times 10^{-6}$ & $6.48 \times 10^{-10}$ \\
$3 \times 10^{-6}$ & $6.48 \times 10^{-9}$ \\
$4 \times 10^{-6}$ & $5.912 \times 10^{-7}$ \\
$5 \times 10^{-6}$ & $2.649 \times 10^{-6}$ \\
$6 \times 10^{-6}$ & $1.0186 \times 10^{-5}$ \\
$7 \times 10^{-6}$ & $2.5254 \times 10^{-5}$ \\
\hline \hline
\end{tabular}

$\omega_{0}(=0.05)$ and the field strength $E_{0}(=0.1)$ of the multisheeted Sauter pulse considered in this article, the Keldysh parameter $\gamma=m \omega / e E=0.5$. Therefore, for the pulse without any chirp, the pair production takes place largely through the tunneling mechanism. On the other hand, in the presence of quadratic chirp, the value of $\gamma$ increases with the increase in the effective frequency in the noncentral part of the pulse. When the chirp is sufficiently strong, the multiphoton process also begins to contribute to the pair production, resulting in the enhancement of the pair production rate. A somewhat similar enhancement in pair production for a probe photon propagating through a polarized ultrashort laser pulse was reported in the analytical work of Titov et al. [43]. The observed enhancement in the pair production, which takes place through the multiphoton process for the system considered therein, was ascribed to the presence of higher frequencies than the central frequency of the laser pulse in its power spectrum. However, in this case, the higher frequencies arise due to the finiteness of the temporal pulse envelope.

\section{CONCLUSION}

To summarize, we have studied FIPT in the presence of a time-dependent Sauter pulse by converting the QKE for the single-particle distribution function into a set of two nonlinear coupled equations describing the evolution of the modulus $|\Phi(\bar{p}, t)|$ and phase $\psi(\bar{p}, t)$ of the complex order parameter associated with the phase transition. The dynamics of $|\Phi(\bar{p}, t)|$ is governed by the product $\left(E(t) / \omega^{2}(\bar{p}, t)\right) \cos \psi(\bar{p}, t)$, and that of $\psi(\bar{p}, t)$ is dictated by two competing terms in the form of $2 \omega(\bar{p}, t)$, the dynamical energy gap, and $\left(E(t) / \omega^{2}(\bar{p}, t)\right)(\sin \psi(\bar{p}, t) /$ $|\Phi(\bar{p}, t)|$ ), under the approximation $|\Phi(\bar{p}, t)| \ll 1$ (which indeed is true for all the cases considered here). With the help of these coupled equations, we have been able to shed light on the origin of different evolution stages reported in Refs. [36,37,40] for the single-sheeted Sauter pulse and multisheeted Gaussian pulse. We find that in such cases $\psi(\bar{p}, t)$ remains nearly constant or varies very slowly with time in the beginning, as the competing terms governing the evolution nearly cancel out. Consequently, $\cos \psi(\bar{p}, t)$ varies slowly with the timescale comparable to that of the external electric field, thereby resulting in the QEPP stage of FIPT, wherein the evolution of $|\Phi(\bar{p}, t)|$ follows the profile of $E(t) / \omega^{2}(\bar{p}, t)$. The slow variation of $\psi(\bar{p}, t)$ is followed by its abrupt steep increase. This makes $\cos \psi(\bar{p}, t)$ oscillate much more rapidly than $E(t) / \omega^{2}(\bar{p}, t)$. In this temporal regime, known as the transient stage, the evolution of $|\Phi(\bar{p}, t)|$ is modulated by the rapid oscillation of $\cos \psi(\bar{p}, t)$. With further increase in time, electric field strength becomes vanishingly small. As a result, the equations describing the dynamics of $|\Phi(\bar{p}, t)|$ and $\psi(\bar{p}, t)$ are decoupled. $|\Phi(\bar{p}, t)|$ becomes a constant, and the dynamics of $\psi(\bar{p}, t)$ is governed by the dynamical energy gap $2 \omega(\bar{p}, t)$. 
$|\Phi(\bar{p}, t)|$ is related to the single-particle distribution function, which gives the number of created particles (antiparticles) for asymptotic times, which in this case is the REPP stage of evolution of the order parameter. In the QEPP stage, the phase $\psi(\bar{p}, t)$ gives a measure of coherence in the particle-antiparticle correlation function in the vacuum state. The oscillations of $\psi(\bar{p}, t)$ in the transient stage of evolution signify "dephasing," which results in the loss of coherence of the correlation function, which subsequently results in the creation of "real" pairs. The real part of the order parameter, $u(\bar{p}, t)=$ $|\Phi(\bar{p}, t)| \cos \psi(\bar{p}, t)$, gives vacuum polarization; whereas the imaginary part, $v(\bar{p}, t)=|\Phi(\bar{p}, t)| \sin \psi(\bar{p}, t)$, represents the corresponding counterterm. It is found that in the QEPP stage $u(\bar{p}, t) \ll v(\bar{p}, t)$. It is in the transient stage that $u(\bar{p}, t)$ and $v(\bar{p}, t)$ are nearly equal. Thus, the process of pair production is associated with the sufficient weakening of the "counterterm" of the vacuum polarization.

In the presence of frequency chirp, the QEPP stage of evolution of $|\Phi(\bar{p}, t)|$ gets far more complex with faster and irregular oscillation, which is attributed to the complexity in $E(t) / \omega^{2}(\bar{p}, t)$. The most striking effect of quadratic frequency chirp is the appearance of a novel stage in the otherwise QEPP stage of the evolution of $|\Phi(\bar{p}, t)|$, located symmetrically opposite to the transient stage from the pulse center. The onset of the pretransient region, as in the transient stage, is accompanied by rapid oscillations in $\cos \psi(\bar{p}, t)$, which in turn arise because of the dominance of the $\left(E(t) / \omega^{2}(\bar{p}, t)\right)(\sin \psi(\bar{p}, t) /|\Phi(\bar{p}, t)|)$ term over the $2 \omega(\bar{p}, t)$ term in the dynamics of $\psi(\bar{p}, t)$. With sufficient increase in the quadratic chirp, the pretransient and transient stages move closer to the pulse center. The rapid oscillations in $\cos \psi(\bar{p}, t)$ continue uninterrupted from the pretransient to the transient stages. An early occurrence of the transient stage leads to the REPP stage with a higher value of $|\Phi(\bar{p}, \infty)|$, resulting thereby in the enhancement of the pair production rate. It may be possible to relate the rich dynamical features of the pretransient and transient stages to the vacuum polarization current, which is in principle an experimentally measurable quantity. Here the polarization current density $j_{p o l}(\bar{p}, t)=\mathrm{geV}^{3} \int[d p] P_{p} / \omega(\bar{p}, t) u$, where $u=|\Phi(\bar{p}, t)| \cos (\psi(\bar{p}, t))$, which depends on the phase of the complex order parameter [44]. Therefore, the evolution of the oscillating phase is an important parameter to study, especially in the pretransient and transient regions.

Measuring pulse parameters of ultrashort and ultraintense laser pulses is a challenging task. Experiments based on the QED effects have been suggested to measure the carrier envelope phase $[45,46]$, the relative content of e- and h-waves [47], the relative phase [48], and the temporal envelope [42] of the counterpropagating pulses. Our studies suggest that enhancement of the pair production rate and polarization current can be used to measure the quadratic chirp parameter of ultrashort pulses.
[1] W. Heisenberg and H. Euler, Z. Phys. 98, 714 (1936).

[2] F. Sauter, Z. Phys. 69, 742 (1931).

[3] J. Schwinger, Phys. Rev. 82, 664 (1951).

[4] A. I. Nikishov, Sov. Phys. JETP 30, 660 (1970).

[5] N. Narozhny and A. Nikishov, Yad. Fiz. 11, 1072 (1970).

[6] T. Tajima and G. Mourou, Phys. Rev. ST Accel. Beams 5, 031301 (2002).

[7] T. Heinzl and A. Ilderton, Eur. Phys. J. D 55, 359 (2009).

[8] G. V. Dunne, Eur. Phys. J. D 55, 327 (2009).

[9] I. C. E. Turcu et al., Romanian Reports in Physics 68, S145 (2016).

[10] http://www.eli-np.ro/.

[11] J. Andruszkow et al., Phys. Rev. Lett. 85, 3825 (2000).

[12] C. Bula et al., Phys. Rev. Lett. 76, 3116 (1996).

[13] D. L. Burke et al., Phys. Rev. Lett. 79, 1626 (1997).

[14] E. Brezin and C. Itzykson, Phys. Rev. D 2, 1191 (1970).

[15] S. P. Kim and D. N. Page, Phys. Rev. D 73, 065020 (2006).

[16] C. K. Dumlu, Phys. Rev. D 82, 045007 (2010).

[17] C. K. Dumlu and G. V. Dunne, Phys. Rev. D 83, 065028 (2011).

[18] A. M. Fedotov, E. G. Gelfer, K. Yu. Korolev, and S. A. Smolyansky, Phys. Rev. D 83, 025011 (2011).

[19] S. P. Kim and D. N. Page, Phys. Rev. D 65, 105002 (2002).
[20] S. P. Kim and C. Schubert, Phys. Rev. D 84, 125028 (2011).

[21] S. P. Kim, H. K. Lee, and Y. Yoon, Phys. Rev. D 78, 105013 (2008).

[22] W. Y. P. Hwang and S. P. Kim, Phys. Rev. D 80, 065004 (2009).

[23] S. P. Gavrilov and D. M. Gitman, Phys. Rev. D 53, 7162 (1996).

[24] H. T. Elze and U. Heinz, Phys. Rep. 183, 81 (1989).

[25] F. Hebenstreit, R. Alkofer, and H. Gies, Phys. Rev. D 82, 105026 (2010).

[26] I. Bialynicki-Birula, P. Górnicki, and J. Rafelski, Phys. Rev. D 44, 1825 (1991).

[27] S. Schmidt, D. Blaschke, G. Röpke, A. Prozorkevich, S. Smolyansky, and V. Toneev, Phys. Rev. D 59, 094005 (1999).

[28] S. M. Schmidt, D. Blaschke, G. Röpke, S. A. Smolyansky, A. V. Prozorkevich, and V. D. Toneev, Int. J. Mod. Phys. E 07, 709 (1998).

[29] D. B. Blaschke, A. V. Prozorkevich, G. Röpke, C. D. Roberts, S. M. Schmidt, D.S. Shkirmanov, and S. A. Smolyansky, Eur. Phys. J. D 55, 341 (2009).

[30] F. Hebenstreit, Ph.D. thesis, University of Graz, 2011.

[31] Y. Kluger, E. Mottola, and J. M. Eisenberg, Phys. Rev. D 58, 125015 (1998). 
[32] Y. Kluger, J. M. Eisenberg, B. Svetitsky, F. Cooper, and E. Mottola, Phys. Rev. Lett. 67, 2427 (1991).

[33] J. Rau, Phys. Rev. D 50, 6911 (1994).

[34] A. V. Filatov, A. V. Prozorkevich, S. A. Smolyansky, and V. D. Toneev, Phys. Part. Nucl. 39, 886 (2008).

[35] S. Coleman, J. Math. Phys. (N.Y.) 7, 787 (1966).

[36] S. A. Smolyansky et al., Ultrametric Anal. Appl. 4, 319 (2012).

[37] S. A. Smolyansky, A. D. Panferov, D. B. Blaschke, L. Juchnowski, B. Kämpfer, and A. Otto, Russ. Phys. J. 59, 1731 (2017).

[38] XFEL home page, https://www.xfel.eu.

[39] https://www.mathworks.com/help/matlab/ref/ode113.html.

[40] D. B. Blaschke, B. Kämpfer, A. D. Panferov, A. V. Prozorkevich, and S. A. Smolyansky, Contrib. Plasma Phys. 53, 165 (2013).
[41] F. Hebenstreit, R. Alkofer, and H. Gies, Phys. Rev. D 82, 105026 (2010).

[42] C. Banerjee and M. P. Singh, arXiv:1807.06951.

[43] A. I. Titov, H. Takabe, B. Kämpfer, and A. Hosaka, Phys. Rev. Lett. 108, 240406 (2012).

[44] S. Smolyansky, D. V. Churochkin, V. V. Dmitriev, A. D. Panferov, B. Kämpfer, S. Bondarenko, V. Burov, and A. Malakhov, EPJ Web Conf. 138, 06004 (2017).

[45] F. Mackenroth, A. Di Piazza, and C. H. Keitel, Phys. Rev. Lett. 105, 063903 (2010).

[46] C. Banerjee and M. P. Singh, Eur. Phys. J. D 72, 4 (2018).

[47] C. Banerjee and M. P. Singh, J. Exp. Theor. Phys. 125, 12 (2017).

[48] C. Banerjee, M. P. Singh, and A. M. Fedotov, Phys. Rev. A 98, 032121 (2018). 